\title{
Telenovela Aliados, educación en valores y activismo juvenil en Argentina
}

\author{
Soap opera Allies, values education and \\ youth activism in Argentina
}

\author{
SOLER-AZORÍN, Laura \\ Doctoranda de Filología Hispánica \\ Universidad de Alicante (España) \\ laura.soler@ua.es
}

Recibido: 10 de marzo 2015

Aceptado: 10 de abril 2015

\begin{abstract}
Resumen
La telenovela juvenil Aliados marca un punto de inflexión en la ficción argentina de estas características por su carácter innovador desde la perspectiva del concepto de emisión multiplataforma así como por el carácter comprometido y de denuncia social con el que se pretende, a través de la ficción, que los jóvenes tomen conciencia de los principales problemas que les afectan.
\end{abstract}

\begin{abstract}
The teen sopa opera Allies marks a turning point in Argentina fiction these features for its innovation from the perspective of the concept of broadcast multiplatform as well as the commitment and social criticism character with which it is intended, through fiction, that young people aware of the main problems that affect them.
\end{abstract}

Palabras Clave: telenovela, valores, juventud, emisión multiplataforma.

Key Words: soap opera, values, youth, broadcast multiplatform. 


\section{Introducción}

La telenovela juvenil Aliados es una producción argentina de Telefe, Cris Morena Group y Fox, con un total de 40 capítulos para televisión en dos temporadas, en la primera de ellas, emitida durante 2013, constó de 23 capítulos, mientras que en la segunda, emitida durante 2014, tuvo sólo 17 capítulos.

Esta producción fue dirigida por Carlos Luna, Mariano De María, Mauro Scandolari y Flavio Rondelli, cuya idea original fue de Cris Morena y el guión de Leandro Calderone. Cuenta con un extenso elenco de actores jóvenes protagonizada por Peter Lanzani y Oriana Sabatini.

Pero lo importante de esta producción televisiva es que, por encima de la temática que trata, logra enganchar y entretener al público, principal función de un producto audiovisual de estas características puesto que si este objetivo no se logra el resto carecen de sentido.

Por ello, observamos en diferentes críticas (Ramos, 2013; Guillén, 2013) en relación a los primeros capítulos donde se indica que, a pesar de sus "muchos lugares comunes y diálogos trilladísimos (...) también cuenta con un elenco de jóvenes actores con talento y un buen ritmo narrativo, haciendo que los capítulos se pasen volando" (Ramos, 2013). Se añade, además, que resulta "imposible no conectar emocionalmente con los personajes" (Ramos, 2013).

En otra de las críticas (Guillén, 2013) a esta telenovela titulada "105 días para el fin del mundo aliados, una telenovela juvenil multiplataforma", encontramos una interesante reflexión acerca de esa producción, precisamente el primer capítulo:

Lo que me ha sorprendido al ver el primer capítulo es que, a pesar de mantener la estética de grabación de una telenovela, tiene una buena post producción de efectos especiales y bonitas cámaras lentas que ayudan a la narración de la historia (Guillén, 2013).

Por todo ello, se considera esta reciente producción de 2013, que lleva en emisión la segunda temporada en Argentina durante 2014, como un ejemplo de activismo transmedia en la ficción televisiva y, además, representa un icono de la emisión multiplataforma debido a su carácter pionero en Argentina. Gracias al éxito de público como demuestra la audienciadel primer episodio de Aliados por la pantalla de Telefe (Argentina) donde logró un promedio de 16.3 puntos de rating, con picos de 19 puntos, superando a su competencia, y estableciéndose como el tercer programa más visto del día.

La audiencia a nivel internacional también fue impactante alcanzando un alto nivel de audiencia según FOX y basados en datos de IBOPE, el estreno de la nueva serie Aliados a 
través de la señal para sus audiencias en Latinoamérica, ha logrado un destacado desempeño en ratings. Durante la emisión del primer episodio de Aliados, FOX se posicionó a nivel Panregional (Latinoamérica) en el primer lugar de canales de televisión paga con 2,09 puntos de rating. Asimismo, fue el canal líder de televisión paga en Argentina con 5,56 puntos de rating, Colombia con 1,74 puntos de rating y Perú con 0,98 puntos de rating, mientras que en Guatemala, FOX fue el canal número uno de entretenimiento en televisión paga con 0,79 puntos de rating por este producto televisivo en Argentina según datos de audiencia de la página de Wikipedia dedicada a Aliados. Consideramos que se trata únicamente del inicio de una nueva industria de dimensiones incalculables, por lo menos en telenovelas dirigidas a adolescentes en una población más proclive al uso de las nuevas tecnologías y a la participación a través de ellas en el entretenimiento. Todo ello augura un futuro prometedor en producciones de estas características.

\section{Antecedentes}

\subsection{El éxito de la telenovela juvenil. Sus características}

Las telenovelas de corte juvenil están dirigidas específicamente al público adolescente y en ella los guionistas tratan de manera prioritaria las inquietudes propias de esta franja de edad al ser ellos mismos los protagonistas y, por ende, los propios seguidores televisivos de la misma (Soler Azorín, 2011). Este subgénero televisivo dirigido específicamente a esta franja de edad de 12 a 16 años aproximadamente es frecuente especialmente en un principio en Venezuela, posteriormente en México y en los últimos años en Argentina, principales países productores de esta temática, tal como indica (Gutiérrez, 2009):

La oferta televisiva latinoamericana del último decenio se ha caracterizado por una acelerada producción de programas que buscan la participación activa de las y los jóvenes, tal es el caso de las telenovelas.(...) En México, por ejemplo, la creciente oferta de telenovelas dirigidas a una audiencia juvenil incluye, entre otras: Pobre juventud (1987), Quinceañera (1988), Dulce desafío (1989), Muchachitas (1991), Mágica juventud (1992), Agujetas de color rosa (1994), Amigas y rivales (2001), El juego de la vida (2001) y Clase 406 (2002). Esta última, nominada a los Shine Awards.

Otro de los países pioneros en este ámbito fue Venezuela, tal como se indica en el mismo artículo:

En Venezuela, de los mismos productores y escritores de Por estas calles, surgen dos éxitos juveniles posteriores: A todo corazón (1997), que arrasó con 
el rating en Venezuela, Centroamérica, Estados Unidos, Bolivia y Chile y Así es la vida (1998).

Otro ejemplo destacado a tener en cuenta es el éxito obtenido con la argentina Rebelde Way, producida en 2002, de la productora, actriz y guionista de televisión Cris Morena, seudónimo de María Cristina De Giácomi creadora también de Aliados y de otros éxitos en Argentina, como $B$ y B, Casi Ángeles, Chiquititas, Alma Pirata, Floricienta, Amor mío, Rincón de Luz y Rebelde Way. Ésta última versa sobre el día a día en un colegio de élite de este país y tuvo un éxito tal que pronto se realizó la versión mexicana de la misma, llamada únicamente Rebelde producida en 2006 y que triunfó aún más, si cabe. Tal como se indica en esta monografía acerca de esta producción:

Rebelde es una telenovela del subgénero juvenil producida por Televisa. Ha sido un éxito avasallador, muchos la han calificado como un fenómeno que hasta la presente no ha parado. No sólo es una ficción que un medio produjo, pues ha tenido tal trascendencia e importancia que sin duda será recordada en la historia de la televisión por ser la más popular y al parecer la más influyente en la moda, habla, el comportamiento y búsqueda de identidad de los jóvenes latinoamericanos de la primera década del siglo XXI. ${ }^{1}$

Con esta producción se reinventó y actualizó la narrativa mexicana en este ámbito, puesto que, tal como se indicaba en esta crítica $^{2}$, la temática juvenil en Televisa, estaba en sus horas más bajas:

Las telenovelas juveniles son un género extinto dentro de la programación de Televisa en El Canal de las Estrellas. Ya no tienen horario fijo dentro de la programación y ya no tienen un flujo de desarrollo como en otras épocas. Ahora las telenovelas juveniles se están produciendo en coproducción con canales de nicho como Nickelodeon y Disney.

Como hemos señalado, este subgénero adolescente en México se reactivó enormemente gracias a la adaptación de la telenovela argentina en el país azteca, rebautizada como Rebelde, tal y como podemos comprobar (Lewkowicz, 2014):

The popularity of Rebelde (Rebel, Televisa, Mexico, 2004-2006), within the teen telenovela subgenre, indicates one telenovela that has successfully maintained

\footnotetext{
${ }^{1}$ Disponible en [http://www.monografias.com/trabajos-pdf5/rebelde-propuesta-muy-conservadora-joveneslatinoamericanos/rebelde-propuesta-muy-conservadora-jovenes-latinoamericanos.shtml]

${ }^{2}$ Disponible en [http://novelalounge.com/nn/2012/04/02/10-telenovelas-juveniles-para-la-historia]
} 
the reach of the Mexican telenovela industry, within this new market context. It is argued that Rebelde's investment in developing a form of [sub] cultural proximity for the transnational youth market breathes new life into the classic Mexican telenovela by challenging its traditional and exclusionary narrative in unprecedented ways.

\subsection{La telenovela dirigida a adolescentes en Argentina}

Este tipo de producciones tienen una larga trayectoria y tradición en este país gracias a Cris Morena, pionera en este ámbito, principal creadora y productora de estas 'tiras' específicas para adolescentes siendo la verdadera responsable de su éxito internacional debido a su mérito personal indiscutible, como se verá más adelante.

Sin embargo, en este país del cono sur existen otras producciones de tema juvenil que han alcanzado un gran éxito nacional e internacional y que no están vinculadas a Cris Morena sino a otros productores y canales de televisión. Un buen ejemplo de ello es Patito feo, una mezcla del cuento tradicional del mismo nombre de Hans Christian Andersen y de La Cenicienta de los Hermanos Grimm, una telenovela juvenil que alcanzó un éxito incalculable a nivel internacional con gira de conciertos incluida alrededor del mundo de su protagonista Laura Esquivel, ganadora de un Martín Fierro en 2007 y nominada a un Emmy en $2008^{4}$. Con Patito feo se comercializaron además todo tipo de productos transmedia relacionados con esta ficción, hasta el punto que, si bien sus dos temporadas retransmitieron en los años 2007-2008, todavía a día de hoy en España se encuentran estos artículos que gozan de gran aceptación.

$\mathrm{Si}$ de ficciones juveniles se trata resulta imprescindible mencionar la telenovela juvenil Violetta, puesto que ha sido el éxito más importante de estas características en los últimos años, consiguiendo el premio Martín Fierro 2013 a la mejor producción juvenil del año anterior. Hay que tener en cuenta que estas producciones fueron retransmitidas en el Canal 13 que es el principal competidor de Telefe, los dos principales canales de televisión abierta en Argentina. Dada la indiscutible vinculación de Cris Morena con Telefe, al haber estado casada con Tomás Yankelevich, director de contenidos globales de la cadena Telefe, es por lo que Canal 13 debió esforzarse en crear productos de calidad juvenil que pudieran competir con el sello de calidad y éxito que supone la firma de Cris Morena. En la actualidad se ha logrado un alto nivel en las producciones de estas características dirigidos a adolescentes

\footnotetext{
${ }^{3}$ Los Martín Fierro son premios de radio y televisión de la Argentina, organizados por la Asociación de Periodistas de la Televisión y la Radiofonía Argentinas (APTRA).

${ }^{4}$ Disponible en [www.elpais.com/diario/2009/10/21/radiotv/1256076001_850215.html]
} 
en los dos canales de televisión privada más importantes de este país, Canal 13 y Telefe. Por ello, han conseguido así, desbancar a México en la temática juvenil de las telenovelas donde las preocupaciones y vivencias de los adolescentes en un ambiente musical es aquello que de forma destacada convierte a estas producciones en una garantía de éxito y calidad.

\section{Discusión}

\subsection{Cris Morena productora de Aliados, como eje fundamental del éxito}

Cris Morena lidera hace más de 18 años las producciones orientadas al segmento infantojuvenil. Sus productos marcan estilo y tendencias a seguir por otras cadenas ${ }^{5}$. Tal como se indica en el artículo (Llahí, 2006), esta productora marcó una nueva visión de la ficción infanto-juvenil en Argentina, hasta el punto que creó marca:

para arribar a lo que podríamos llamar "el boom Cris Morena", que comienza con Rejugadísimo en 1994 para continuar con Chiquititas, ciclo de gran aceptación en 1995 y desde el año pasado con Floricienta, su nuevo programa de televisión. Los estereotipos que generan estos programas televisivos, en la mayoría de los casos gozan del beneplácito de los padres

Uno de los casos más significativos es la telenovela de producción argentina Chiquititas, cuya historia acerca de la vida cotidiana de un orfanato de niños fue un fenómeno sin precedentes en la televisión argentina, siendo retransmitida ininterrumpidamente y con enorme repercusión social desde los años 1995 hasta 2001. Tiempo después, concretamente en el 2006, se televisó otra tanda de capítulos, también con mucho éxito. Es interesante el fenómeno popular de Chiquititas por su continuidad en el tiempo y porque prosiguió años después de retirada la emisión sin que sus antiguos seguidores la hubiesen olvidado. Pero lo más llamativo fue el eco que supuso más allá de la televisión; como buen fenómeno de cultura de masas, se realizaron los primeros productos transmedia, mientras se efectuaban representaciones teatrales con el argumento y protagonistas de la misma, obteniendo un éxito rotundo.

Además de ello, se editó una revista sobre la serie y se realizó una película titulada Rincón de luz, que seguía la estela argumental de la misma con muchos de sus protagonistas bajo la supervisión de Cris Morena (Soler, 2011).

\footnotetext{
${ }^{5}$ Disponible en [www.crismorenagroup.com.ar/espanol-11.html]
} 
Tal como se indica en la publicación de las actas /I Jornadas nacionales de Investigación y Crítica teatral (Goldfinger, 2012):

Las versiones de Chiquititas en el teatro tuvieron gran aceptación por parte del público, comienza con la creación de su exitoso Chiquititas. Se trataba de un programa diario sobre niñas huérfanas que residen en un hogar llamado Rincón de Luz. La tira salía del formato tradicional de programas de animación y se presentaba en código de novela para niños (...) funciones a sala llena. Con los elementos mágicos de la serie exacerbados, las historias teatrales solieron devenir en cuentos.

\subsection{Inicios de los productos transmedia en Argentina}

Cris Morena fue de las primeras en implantar los productos transmedia puesto que descubrió el filón que suponían sus producciones comercializando posteriormente en DVD que se vendían y se venden junto al $C D$ con las canciones de éxito que aparecían en sus producciones televisivas. Cris Morena tiene más de 500 canciones registradas con su nombre y, como ocurre en Aliados, siendo la creadora de la idea original y la productora, todo tiene su sello inconfundible con el resto de producciones siguió la estela de merchandising y de productos transmedia cada vez con más fuerza, como se verá más adelante con su última producción.

\subsection{Aliados como punto de inflexión}

Esta telenovela juvenil marca un punto y aparte en la producción ficcional de Cris Morena puesto que si bien todo lo que ella había creado para televisión estaba específicamente dedicado a adolescentes. En ellos trataba temas muy manidos y volvía siempre a los lugares comunes de las telenovelas pero adaptándolos con maestría al público al que iba dirigido. Por ello en Aliados se marca un punto de inflexión sobre la problemática social juvenil actual e intenta realizar una crítica constructiva acerca de estos problemas para concienciar y hacer reflexionar a la juventud.

Fundamentalmente se produce un giro en sus producciones por causa del fallecimiento de su hija Romina de manera repentina, acontecimiento que le causó una gran conmoción. Esta producción está llena de guiños hacia su hija puesto que era un homenaje a ella, tal como señaló Cris Morena en la entrega de los premios Martín Fierro 2014 cuando Aliados recibió el 
premio a la mejor producción juvenil: "Ella (Romina) es mi inspiración y mi luz (Giacommi, 2014)"6.

La línea argumental tiene que ver con la transformación que sufrió Cris cuando falleció su hija y 'su conexión a través de los ángeles para cambiar el mundo'.

Aliados cuenta la historia de jóvenes con diferentes problemáticas que parecen no tener salida por causa del maltrato infantil, el bullyng o la anorexia, la bulimia o la adicción al sexo. La trama se complica cuando llegan unos 'aliados' de otros planetas que tienen un carácter contrapuesto a los jóvenes a los que desean a ayudar. Por ello, se meten en un cuerpo humano para ayudarlos a resurgir y procurar que sean felices, pudiendo así los 'aliados' cumplir con su misión. Tiene sentido, pues, la afirmación que realizó Cris Morena en la entrega de premios en relación al argumento de la misma y además posee muchos guiños con la vida personal de la productora.

Para contextualizar la renovación y el compromiso social como una necesidad vital hay que conocer aquellos detalles de la vida personal de Cris Morena y su familia que, de una manera u otra, aparecen reflejados en la serie: su hija fallecida había sufrido anorexia y bulimia, al igual que Azul, una de las principales protagonistas de la historia. Además, el bar donde se desarrolla parte de la acción se llama 'Vive Ro Bar' y, tal como explicó en los minutos previos al capítulo final de Aliados resulta un acróstico homenaje a su hija: vive Ropor el nombre de Romina, su hija, que está allí presente.

3.4. Narrativa transmedia, merchandising generado a partir de este producto televisivo (CD, DVD, revista semanal y juegos sobre la serie)

Esta tira televisiva comparte con el resto de las producidas por esta carismática mujer, el lanzamiento del CD con las canciones que aparecen al mismo tiempo que se efectúa el estreno de la misma, con un éxito arrollador, hasta el punto que cuando estaba en emisión el octavo capítulo, ya se había convertido en disco de oro. Además, cuando finalizó la primera temporada se pusieron a la venta los DVD con todos los capítulos completos y acompañados de extras con las canciones, el making off $y$ algunas sorpresas más. También se comercializó un DVD exclusivo con los extras.

Con la edición de una revista semanal en la que se tratan todos los temas que interesan a los adolescentes, además de entrevistar y dar a conocer más a fondo a los protagonistas de la serie y un libro que narra la historia de la serie escrito por Leandro Calderone, el guionista, titulado Aliados, entre el cielo y la tierra, casi se cerró el círculo transmedia. Como novedad,

\footnotetext{
${ }^{6}$ Disponible en [http://www.eltrecetv.com.ar/premios-martin-fierro-2014]
} 
se creó el 'Club Aliados'7, que es un club que ofrece diferentes ventajas a los seguidores de la serie, inclusive la posibilidad de participar en encuentros con los protagonistas de la misma.

Sin embargo, no nos podemos olvidar de la tradicional puesta en escena musical de la serie que se produce, siempre, en el Teatro Gran Rex y que ha creado escuela; nos parece importante señalar que en el mayo de 2014 se acaban de poner en venta las entradas para las funciones el próximo mes de julio de 2014 con motivo de las vacaciones del invierno austral.

\subsection{Emisión multiplataforma y ejemplo transmedia}

Esta innovadora producción juvenil presenta características que apenas se han realizado a nivel mundial ya que se observa también un innovador modelo de distribución que funciona de la siguiente manera, según la revista especializada Todo tv news:

Con emisiones prácticamente en simultáneo en TV abierta por medio de Telefe y TV paga a través de Fox (un día después), así como websodios emitidos durante toda la semana que complementan la historia, Aliados es verdaderamente un producto $360(. .$.$) Este es uno de los pocos productos a$ nivel internacional que llevó adelante con éxito el tan mencionado y esperado modelo multiplataforma, que la industria tanto está esperando (Larrea, 2013).

\subsection{Diferentes maneras de conectar con el telespectador}

El Facebook y el Twitter oficial se actualizan constantemente, con una media de una publicación diaria. Se publican diferentes cuestiones: en primer lugar, los adelantos de los capítulos y los websodios; por otro, entrevistas con los protagonistas de la serie y backstage de los actores en su vida cotidiana, como es el caso del vídeo resumen del viaje de una de ellas, concretamente Oriana Sabatini, a Miami.

Por otro lado, los actores promocionan los productos transmedia como el libro, el CD o cualquier otro producto relacionado con la telenovela, acercándolos al comprador más si cabe.

Además, se utilizan las redes sociales como plataforma de concienciación de diversas problemáticas que aparecen en la serie de diferentes formas.

\footnotetext{
${ }^{7}$ Disponible en [http://usuarios.aliados.telefe.com]
} 
Desde mi punto de vista, en este tipo de cambios reside la concienciación social que se pretende lograr en los jóvenes acerca de los problemas cotidianos que les afectan por medio de la empatía de los protagonistas a través de un medio de comunicación de masas como es la telenovela y utilizando como instrumento de enlace las redes sociales.

A través de las mismas se dan mensajes más claros y directos de la realidad, no vinculados solamente a la ficción. Se lanzan mensajes contra el bullyng \#Nobullying como la viralización de fotos en redes sociales con la intención de dañar la imagen de un chico puede considerarse bullyng virtual. Además aparecen vídeos de dos de las protagonistas entrevistándose con una asociación que trabaja con las personas que sufren anorexia y bulimia con la que hablan del porqué de la enfermedad y cómo mejorar su salud.

Además, está estructurado en breves videos de 2 minutos aproximadamente en varias fases, con una información muy concreta en la que también participa un joven que ha superado la enfermedad. Así, de manera atractiva para los jóvenes y muchas veces sin que siquiera sean conscientes de ello, van conociendo y reconociendo aquellas problemáticas sociales que les afectan.

También en la página web existen divididas por secciones las partes "arriba” indicadas donde se refleja todo lo dicho anteriormente, bajo los epígrafes 'mirá', 'sentí' y 'descubrí'. Pero además existe un apartado que titula 'sumá' donde se tratan en forma resumida los temas sociales que, de alguna forma, aparecen en la serie o están de actualidad con motivo de días internacionales, como el Día del Agua o el de Internet.

\subsection{Vinculación de la problemática social que aparece en la serie a través del fenómeno transmediático}

Resulta un paso adelante que en producciones televisivas de esta magnitud se traten temáticas tan complejas y de tanta importancia para los jóvenes, insertadas dentro de una ficción que los atrapa y les mantiene pegados a la pantalla, pero no sólo a ésta, sino al resto de sistemas multimedia que se han puesto en marcha con Aliados, que lo ha hecho mucho más atractivo para este público ya que está muy bien gestionado, a través de mensajes claros y concisos que ayudan a la concienciación social y posterior actuación en este tipo de casuísticas teniendo en cuenta, además, el trabajo en red vinculando el trabajo de las ONG implicadas. Por supuesto, llamando la atención sobre la labor que hacen y como contactar con ellos, en caso de necesitar sus servicios o desear colaborar con las mismas. Un ejemplo muy ilustrativo de ello es el de La Fundación Sí12, que trabaja con personas en situación de vulnerabilidad a lo largo de todo el país, y a través de las redes sociales de la serie donde continuamente se mencionan estas ONG, especialmente cuando se produce una situación de emergencia o un llamamiento de una campaña para colaborar. 
La vinculación del elenco de la serie con la causa es clara puesto que en el inicio de la página de la FundaciónSí, existe un spot de la misma que es una explicación de sus objetivos y un llamamiento a la colaboración con todo el elenco de Aliados como protagonistas, cerrando la propia Cris Morena lanzando mensajes de solidaridad que aparecían la producción televisiva ${ }^{8}$.

\subsection{Propuestas de ocio y comportamientos saludables}

En las redes sociales y en la página web se intentaba ir un poco más allá y se realizaban propuestas de comportamiento saludable, tales como no burlarse de otra persona a través de las redes sociales para evitar el bullyng o propuestas como realizar una quedada con los amigos, libres de pantallas. Son cuestiones básicas de introducción de buenos hábitos según los preceptos de la sociedad actual, dirigidos más específicamente a la juventud y que se pueden ver en el Facebook oficial de Aliados ${ }^{9}$.

\section{Conclusiones}

Teniendo en cuenta lo novedoso de esta producción Aliados por su fuerte apuesta al sistema multiplataforma y multipantalla, debemos decir que ha sido valiente al tratar temas tan complejos que implican de manera directa al telespectador, pudiéndolo convertir en activista social activo. Y si bien a día de hoy carecemos de datos acerca del impacto real que ha causado Aliados en los jóvenes y el hecho de conocer más de cerca las problemáticas que les afectan, como sí ocurrió con otra producción de similares características pero para adultos de este país titulada Montecristo, un amor, una venganza, gracias a la que, tal como se indica en este artículo, se logró que una persona recuperara su identidad gracias a ver una foto suya en la pantalla de la televisión ya que la temática de la telenovela, basada parcialmente en la novela de Dumas, era la desaparición de niños durante la dictadura militar argentina y la lucha de Abuelas de Plaza de Mayo en la recuperación de la identidad perdida (Sueldo, 2012: 180):

The telenovela Montecristo is a communicative phenomenon by which mass media and human rights joined forces to promote current issues within Abuelas de Plaza de Mayo. It incorporated in its plot a traumatic process: a woman findsher real identity, the one who was stolen during the 1970s by the dictatorial government in Argentina. This process displays topics such as memory, justice,

\footnotetext{
${ }^{8}$ Disponible en [http://www.fundacionsi.org.ar] y Disponible en [https://www.youtube.com/watch?v=gXkuqriW1TUsi.org.ar]

${ }^{9}$ Disponible en [www.facebook.com/mundoaliados]
} 
and power in current Argentine society. Montecristo was broadcasted in Argentina during 2006 and in the United States during 2008-2009, and it represents a change (among others) in Abuelas de Plaza de Mayo approach to their search. This change meant to promote awareness regarding their search for babies and children who were kidnapped during the last Argentine dictatorship. This essay also analyzes the genre telenovela as a cultural production with specific features in a growing transnational cultural market and its position inside the Latin American cultural industry.

Sin embargo, un buen indicador para poder calibrar el éxito de esta serie y su aceptación entre los jóvenes son los altos datos de rating transmedia de los primeros ocho capítulos de la serie, según indica la web oficial de la telenovela ${ }^{10}$.

Desde su estreno, Aliados, en sus primeros ocho capítulos emitidos, había logrado unas cifras sorprendentes ya que la web www.telefe.com/aliados tuvo más de 6 millones de visitas y 29 millones de páginas vistas. Los websodios, con una duración promedio de 7 minutos, han logrado un tiempo de visualización del 95\%. ${ }^{11}$

La telenovela consta de 40 capítulos para televisión que al mismo tiempo se traducen en 280 capítulos para internet con una programación prácticamente diaria para seguir la historia de Aliados, de ahí que haya tan pocos capítulos en TV.

Por otro lado, se debe destacar el dato en referencia a la primera temporada, donde se indica que,"El flujo digital de Aliados no se detiene ahí: en junio fue el programa argentino con más menciones en Twitter (por encima de las 850 mil menciones), mientras que en YouTube el contenido de la ficción ya superó la barrera de las cuatro millones de reproducciones" (Página 12, 2013). ${ }^{12}$

A todo ello le sumamos el alto share que obtuvo durante la primera temporada en televisión, aquí analizada, y que ha mantenido buenos resultados en la segunda temporada emitida en 2014 con más productos transmedia y más implicación todavía del telespectador en la trama. Sin duda resulta una buena muestra de lo que será la televisión del futuro: calidad, emisión multiplataforma, implicación del telespectador y sensibilidad social. Una combinación en aras de un crecimiento prometedor de la ficción.

\footnotetext{
${ }^{10}$ Disponible en [http://www.socialtves.com/105-dias-para-el-fin-del-mundo-aliados-una-telenovela-juvenil-multiplataforma]

${ }_{11}$ Disponible en [http://www.socialtves.com/105-dias-para-el-fin-del-mundo-aliados-una-telenovela-juvenil-multiplataforma]

12 Disponible en [http://www.pagina12.com.ar/diario/suplementos/espectaculos/subnotas/30338-7643-2013-10-27.html]
} 


\section{Bibliografía}

GOLDFINGER, E. Y. (2012): “Teatro-TV: cuando la televisión hace teatro para niños". PRIMER PREMIO - II Jornadas Nacionales de Investigación y Critica Teatral, 5. Disponible en:

http://www.aincrit.org/pdfs/actasiijornadasnacionalesdeinvestigacionycriticateatral2010.pdf\# page $=7$

GUILLÉN, J. (2013): "105 días para el fin del mundo: 'ALIADOS' una telenovela juvenil multiplataforma" en Socialteves.com. Disponible en: http://www.socialtves.com/105-diaspara-el-fin-del-mundo-aliados-una-telenovela-juvenil-multiplataforma/

GUTIÉRREZ, G. A. (2009). Los medios de comunicación en Colombia frente a las demandas de los ciudadanos. Actualidades Pedagógicas, (54), 137-145.

LEWKOWICZ, E. (2014): "Rebel love: Transnational teen TV vs. Mexican telenovela tradition”, en: Continuum, 28, nํㅡㄹ. Págs. 265-280. School of the Arts and Media, University of New South Wales, Sydney, Australia

LLAHÍ, S. (2006): Un acercamiento a la historia del teatro para niños en Buenos Aires. Pensar en Grande para Chicos, Cuadernos del Picadero, (9).

RAMOS, J. (2013): "Primeras impresiones de Aliados" en Todotnv.com. Disponible en: http://www.todotnv.com/primeras-impresiones-de-aliados.html

SOLER AZORÍN, L (2011): Ciudad y telenovela en Latinoamérica. Universidad de Alicante. DEA. (Págs. 37-41 y 98-110).

SUELDO, M. (2012): Studies in Latin American Popular Culture. Volume: 30. Pages: 180-193

\section{Forma de citar este artículo en bibliografías}

SOLER-AZORÍN, L. (2015): "Telenovela Aliados, educación en valores y activismo juvenil en Argentina", en Revista PANGEA, 6, páginas 23 a 35. Red Académica Iberoamericana de Comunicación. Recuperado el de de 2 de: http://revistapangea.org 